\title{
NCBO Overview and Biositemaps
}

Trish Whetzel, PhD

\author{
THE NATIONAL CENTER FOR \\ BIOMEDICAL ONTOLOGY
}




\section{National Center for Biomedical Ontology}

- Mission

- To create software for the application of ontologies in biomedical science and clinical care

- NCBO Partners

- Stanford University - Dr. Mark A. Musen

- Mayo Clinic - Dr. Christopher G. Chute

- University of Buffalo - Dr. Barry Smith

- University of Victoria - Dr. Margaret-Anne Storey 


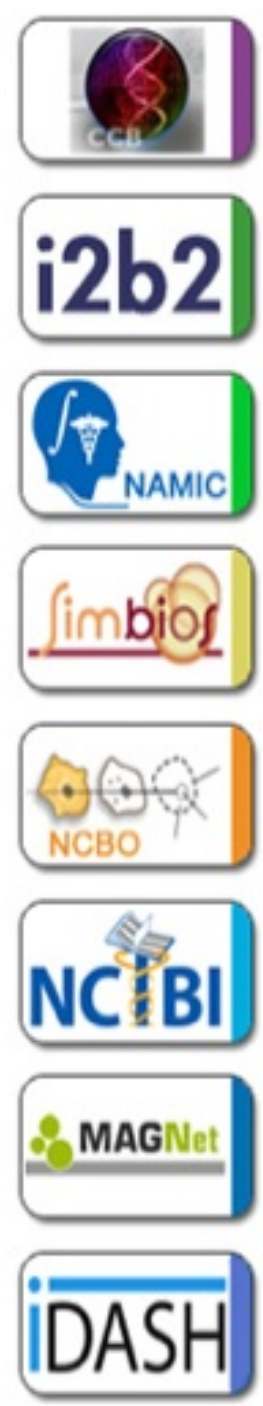

NCBC Centers

Informatics for Integrating Biology and the Bedside

Brigham and Women's Hospital

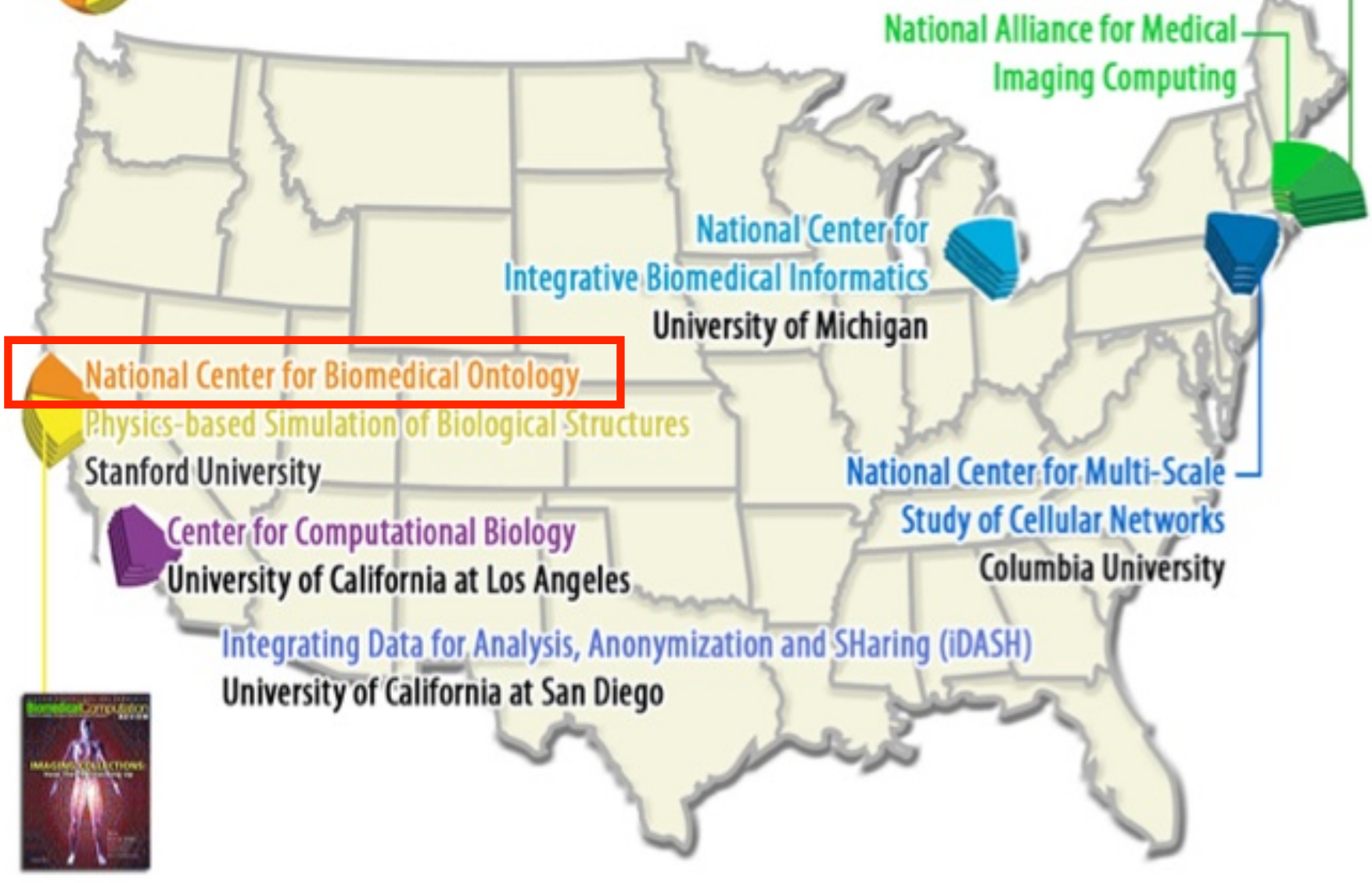

\section{National Centers for Biomedical Computing} (http://www.ncbcs.org) 


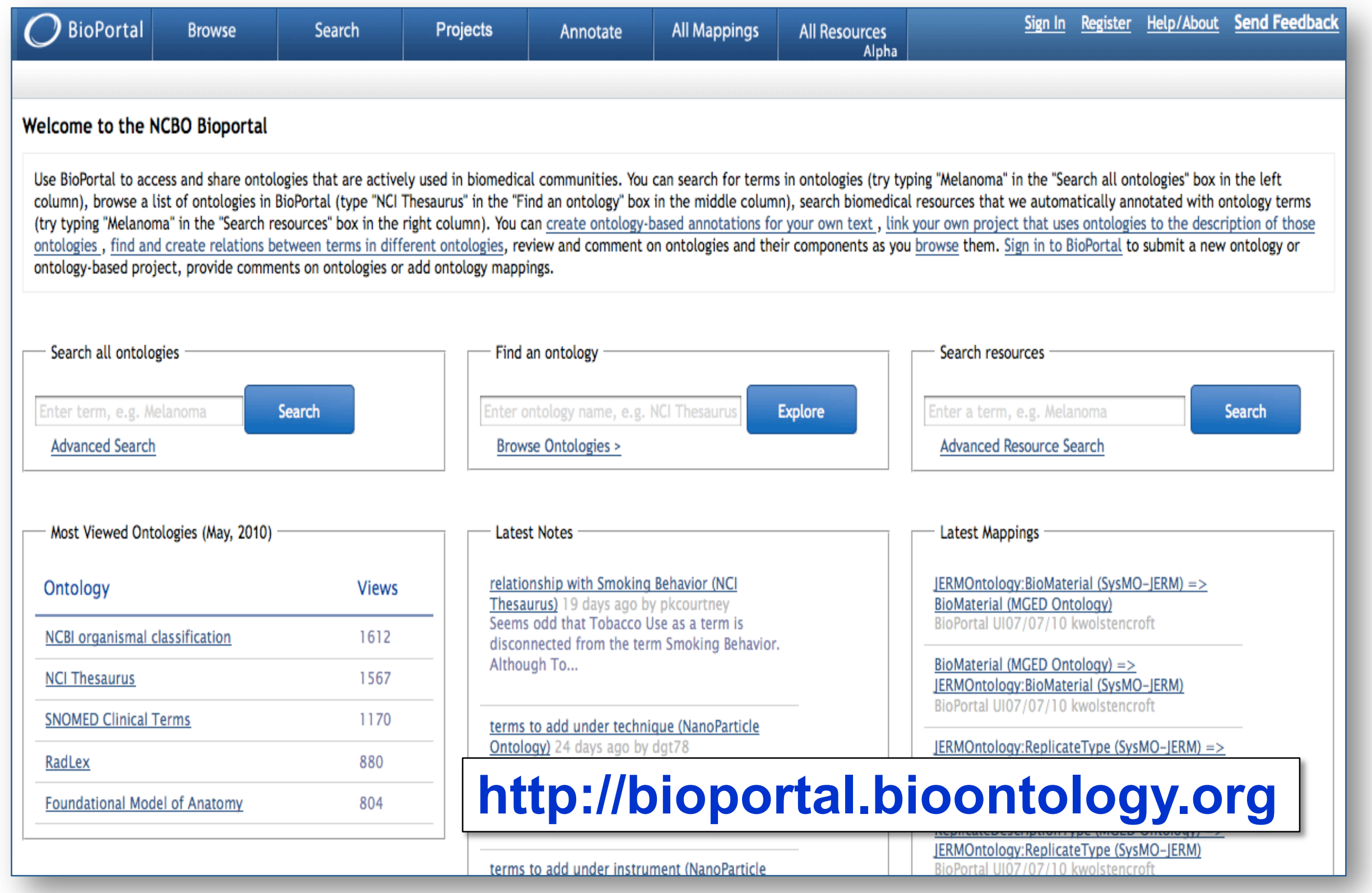




\section{BioPortal}

- Library of Ontologies

- NCBO BioPortal includes Biomedical ontology content, e.g. UMLS terminologies

- Architecture

- Backend includes LexEVS and Protégé

- Web Interface uses Ruby on Rails, Flex, and PHP

- Web services

- REST Web services 


\section{Ontology Web Services}

- Access Ontologies and Views (Value sets)

- Search

- Term

- Hierarchy

- Mapping

- Notes

- Widgets

- Auto-complete

- Visualization

- Create ontology-based annotations

- Annotator

- Ontology Recommender

- Lexicon Builder

- Access ontology-based index of annotations

- Resource Index 


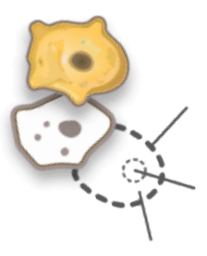

\begin{tabular}{|c|c|c|}
\hline Software & $\begin{array}{c}\text { NCBO } \\
\text { Technology }\end{array}$ & URL \\
\hline ALEX & $\mathrm{W}$ & https://alex.med.nyu.edu/portal/ \\
\hline aTag Generator & $\mathrm{W}$ & http://hcls.deri.org/atag/generator/ \\
\hline BioLit & $\mathrm{O}$ & $\begin{array}{l}\mathrm{http} / / / \text { biolit.ucsd.edu/doc/ } \\
\text { http://protegewiki.stanford.edu/wiki// }\end{array}$ \\
\hline BioPortal Reference Plugin & $\mathrm{O}$ & $\begin{array}{l}\text { BioPortal_Reference_Plugin } \\
\text { http://protegewiki.stanford.edu/wiki/ }\end{array}$ \\
\hline BioPortal Import Plugin & $\mathrm{O}$ & $\begin{array}{l}\text { BioPortal Import Plugin } \\
\text { https://wiki.birncommunity.org/displ }\end{array}$ \\
\hline $\begin{array}{l}\text { BioScholar } \\
\text { cgMDR }\end{array}$ & $\begin{array}{l}\mathrm{O} \\
\mathrm{O}\end{array}$ & $\begin{array}{l}\frac{\text { ay/NEWBIRNCC/BioScholar }}{\text { http://goo.gl/dvsgM }} \\
\text { http://www3.imperial.ac.uk/cisbic/c }\end{array}$ \\
\hline $\begin{array}{l}\text { CISBIC Data Management } \\
\text { eleMAP }\end{array}$ & $\begin{array}{l}\mathrm{O} \\
\mathrm{O}\end{array}$ & $\begin{array}{l}\text { orefacilities/datamanagement } \\
\text { https://victr.vanderbilt.edu/eleMAP/ } \\
\text { http://en.wikipedia.org/wiki/Portal: }\end{array}$ \\
\hline GeneWiki & A & $\begin{array}{l}\text { Gene Wiki } \\
\text { http://sites.google.com/site/icd11rev }\end{array}$ \\
\hline iCAT & $\mathrm{O}$ & $\begin{array}{l}\text { ision/home/icat } \\
\text { http://isatab.sourceforge.net/isacreat }\end{array}$ \\
\hline ISAcreator & $\mathrm{O}, \mathrm{A}$ & $\begin{array}{l}\text { or.html } \\
\text { http://ncmir.ucsd.edu/downloads/jin }\end{array}$ \\
\hline Jinx & $\mathrm{O}$ & X.shtm \\
\hline $\begin{array}{l}\text { Knowledge Egg } \\
\text { Microsoft Word Addin for Ontology }\end{array}$ & W & http://www.kunnskapsegget.no/ \\
\hline Recognition & $\mathrm{O}$ & $\begin{array}{l}\text { http://ucsdbiolit.codeplex.com/ } \\
\text { http://nemo.nic.uoregon.edu/wiki/N }\end{array}$ \\
\hline NEMO Toolkit & $\mathrm{O}$ & EMO_ERP_Analysis_Toolkit \\
\hline Nutritional Phenotype database & $\mathrm{W}$ & $\begin{array}{l}\text { http://www.dbnp.org/ } \\
\text { http://www.bioontology.org/ODIE- }\end{array}$ \\
\hline ODIE & A & project \\
\hline ODiSSea & $\mathrm{O}, \mathrm{A}, \mathrm{RI}$ & http://www.hub.sciverse.com/ \\
\hline OntoCat & $\mathrm{O}$ & http://www.ontocat.org/ \\
\hline Ontological Discovery Environment & A & $\begin{array}{l}\text { http://ontologicaldiscovery.org/ } \\
\text { http://citih.osumc.edu/projects/proje }\end{array}$ \\
\hline openMDR & $\mathrm{O}$ & $\begin{array}{l}\text { ct\&r=1032 } \\
\text { http://www.shigen.nig.ac.jp/rice/ory }\end{array}$ \\
\hline Oryzabase & $\mathrm{W}$ & zabase/top/top.jsp \\
\hline RadLex Tree Browser & $\mathrm{O}, \mathrm{W}$ & $\begin{array}{l}\text { http://www.radlex.org/ } \\
\text { http://digitaleveredelung.dfki.de/ME }\end{array}$ \\
\hline RadSpeech & $\mathrm{W}$ & DICO-Playground/term2.html \\
\hline REDfly & $\mathrm{W}$ & http://redfly.ccr.buffalo.edu/ \\
\hline RightField & $\mathrm{O}$ & $\begin{array}{l}\text { http://www.sysmo-db.org/rightfield } \\
\text { https://sites.google.com/a/s3db.org/s }\end{array}$ \\
\hline S3DB & $\mathrm{O}$ & $\underline{3 \mathrm{db} /}$ \\
\hline SEEK & $\mathrm{O}$ & http://www.sysmo-db.org/seek \\
\hline
\end{tabular}




\section{Biositemaps: From the Beginning}

- The NCBCs originally listed software and data resources on a wiki

- The wiki was not easily scalable or searchable, and was useable only by humans

- And then, Google sitemaps provided a key insight

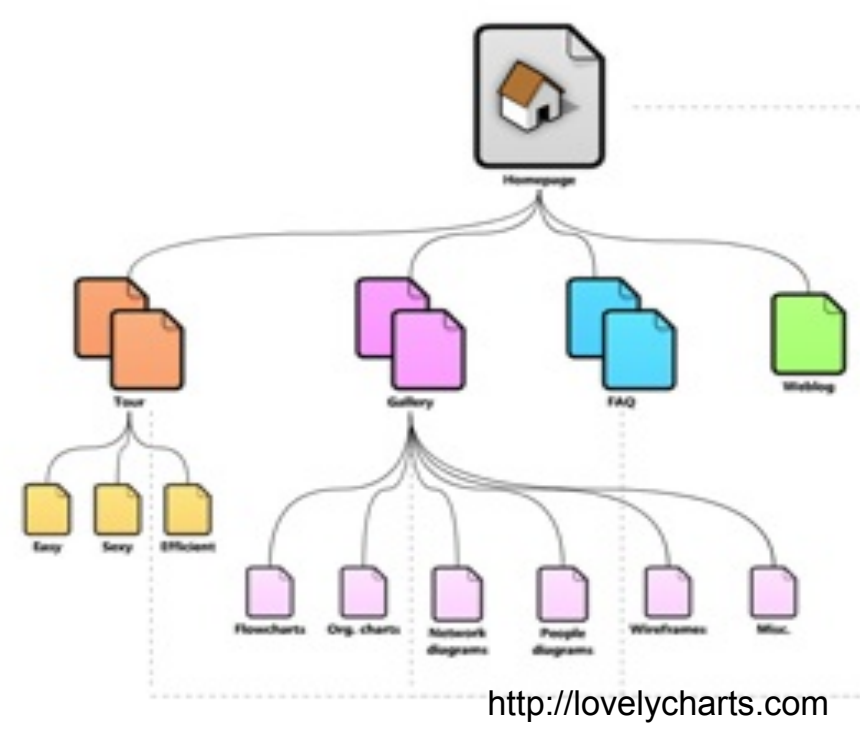




\section{Biositemaps}

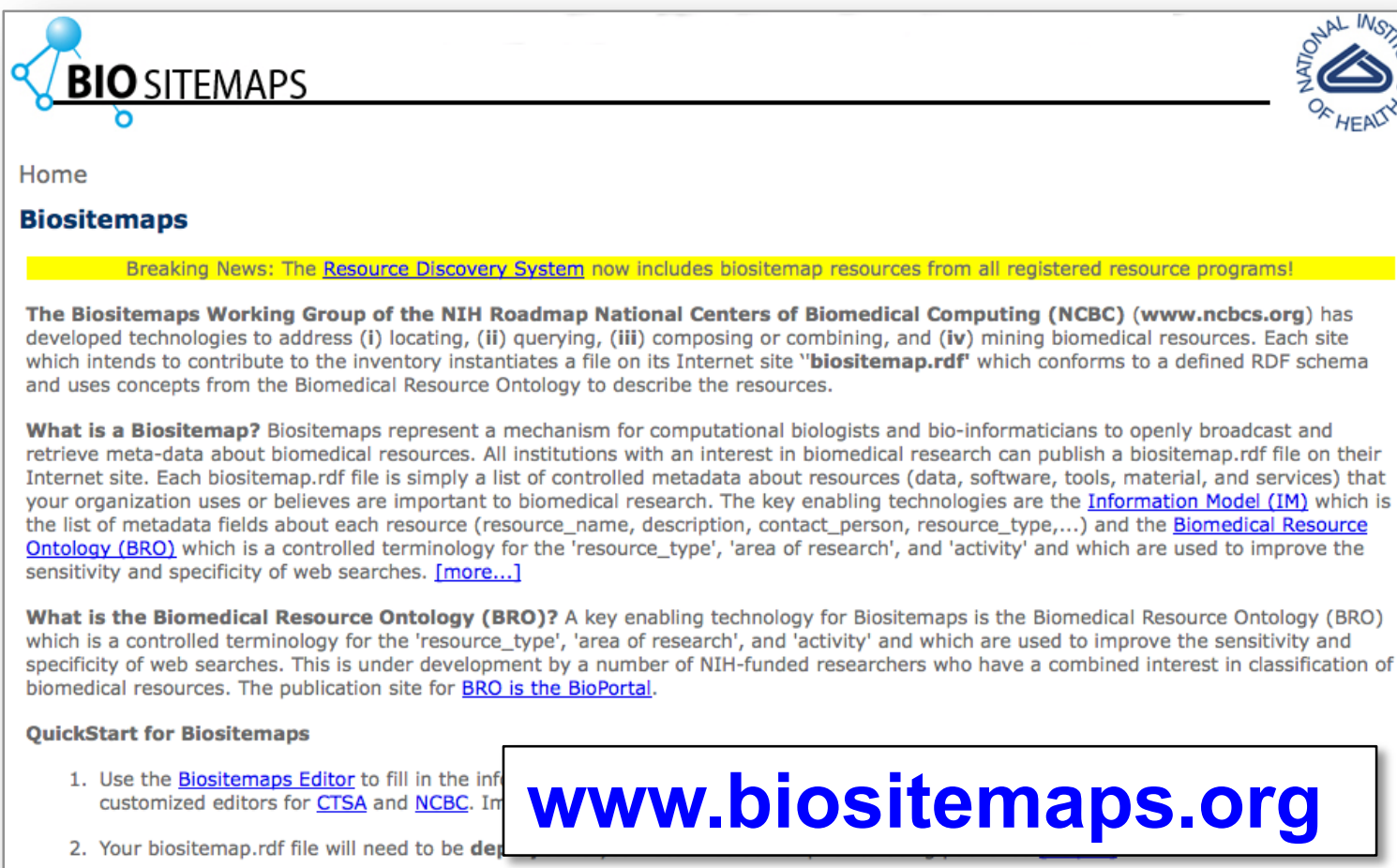

3. Use the Biositemap Registration to publish your biositemap after it has been deployed [help...]

BIOSITEMAPS
- Machine-readable resource associated with a website

- Resources are described in terms of an information model

- Supported by the Biomedical Resource Ontology

- Can be discovered by search engines such as Google 


\section{Biositemaps}

National Centers for Biomedical Computing

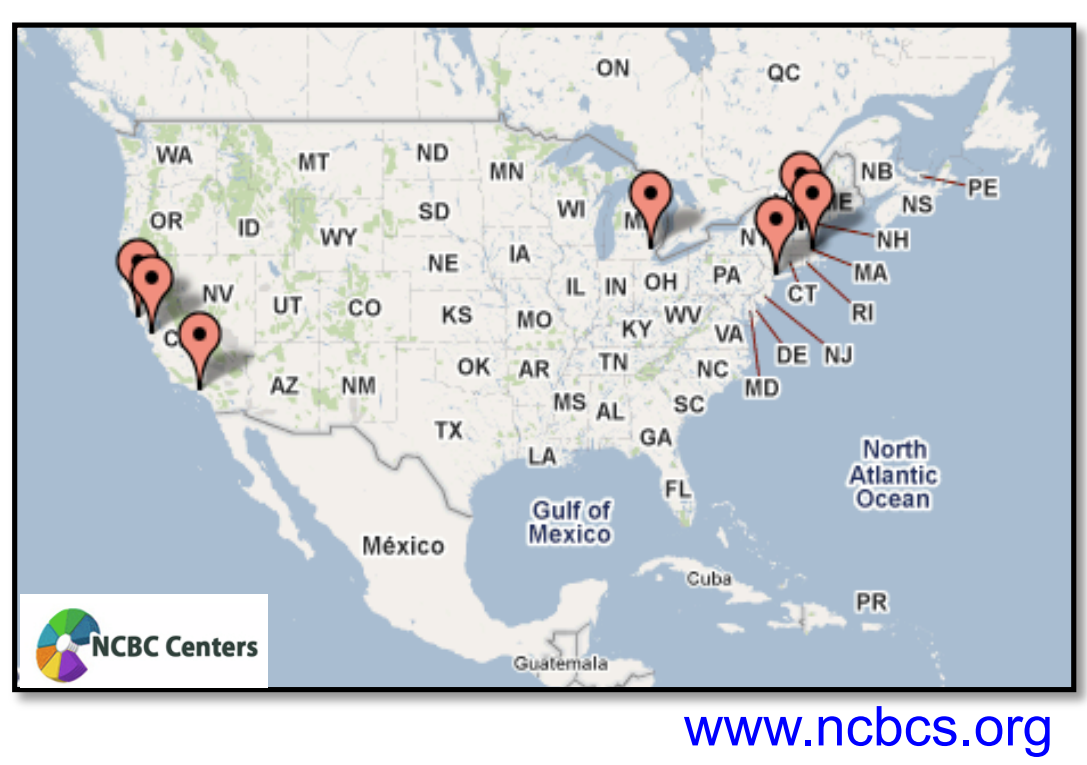

Clinical and Translational Science Awards

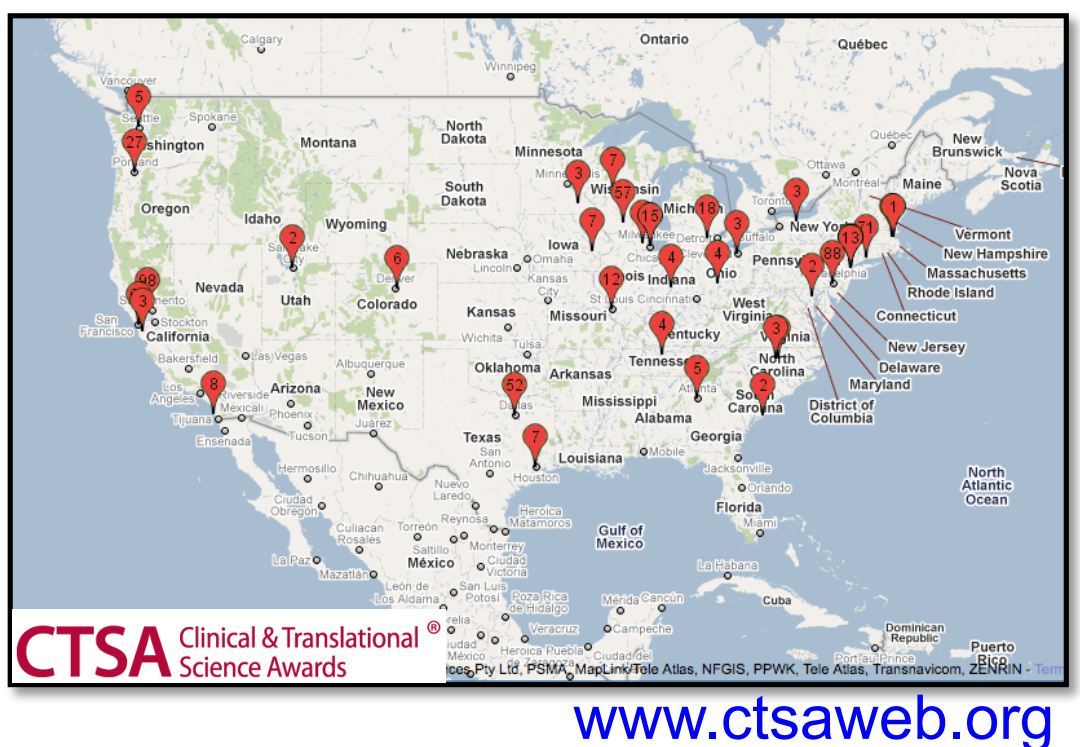




\section{Biositemaps Information Model (v 2.6)}

- Required Properties

- Resource Name

- Organization

- Research Program

- Description

- Resource Type

- URL

- Contact Person

- Biositemap Author 


\section{Biositemaps Information Model}

\section{(v 3.2.1)}

- Required Properties

- Resource Name

- Organization

- Research Program

- Description

- Resource Type

- URL

- Contact Person

- Biositemap Author
- Additional Properties

- Related Areas of Research

- Related Activities

- Publication Identifier 


\section{Biositemaps Editor}

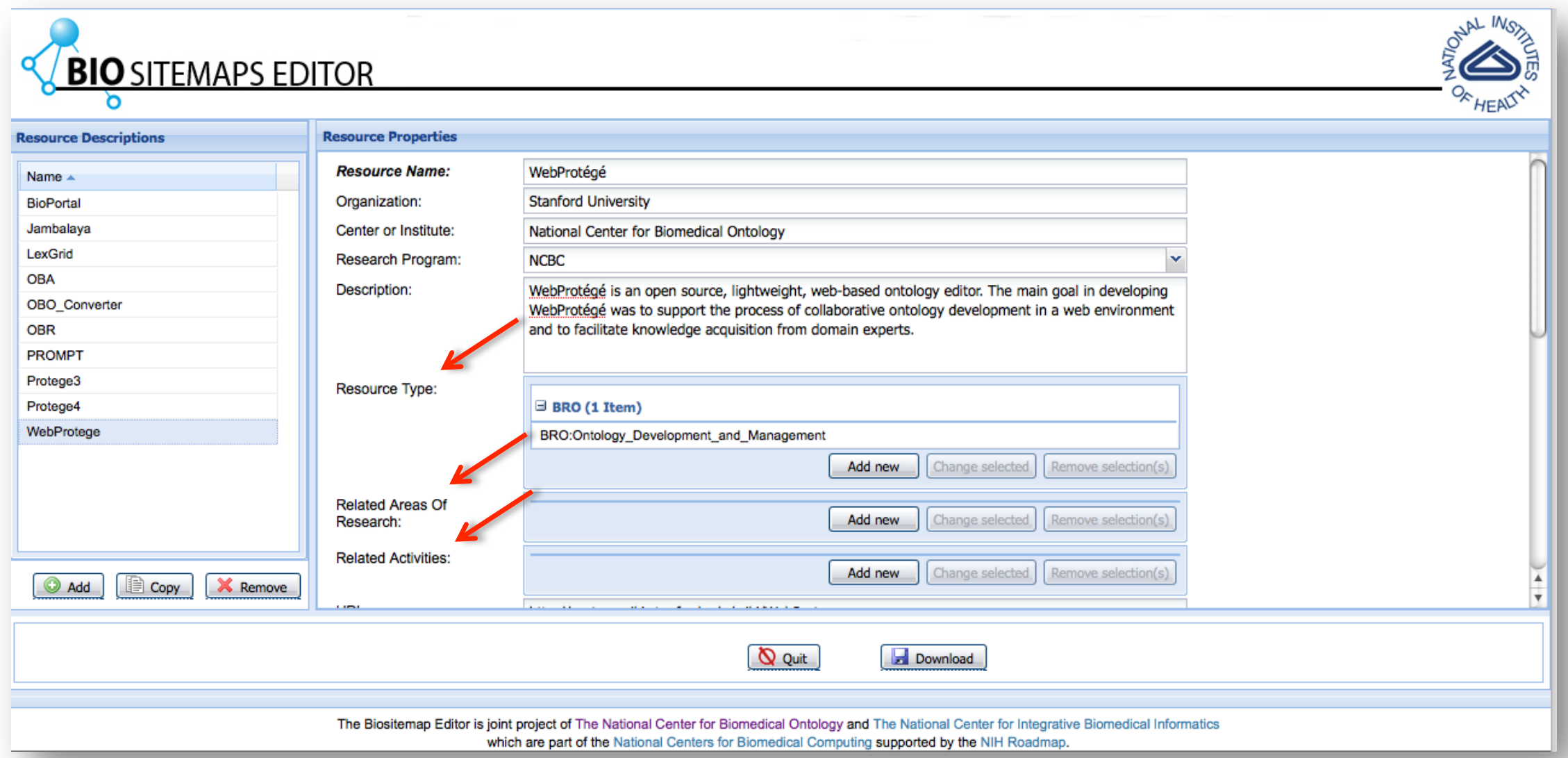

BIOSITEMAPS 


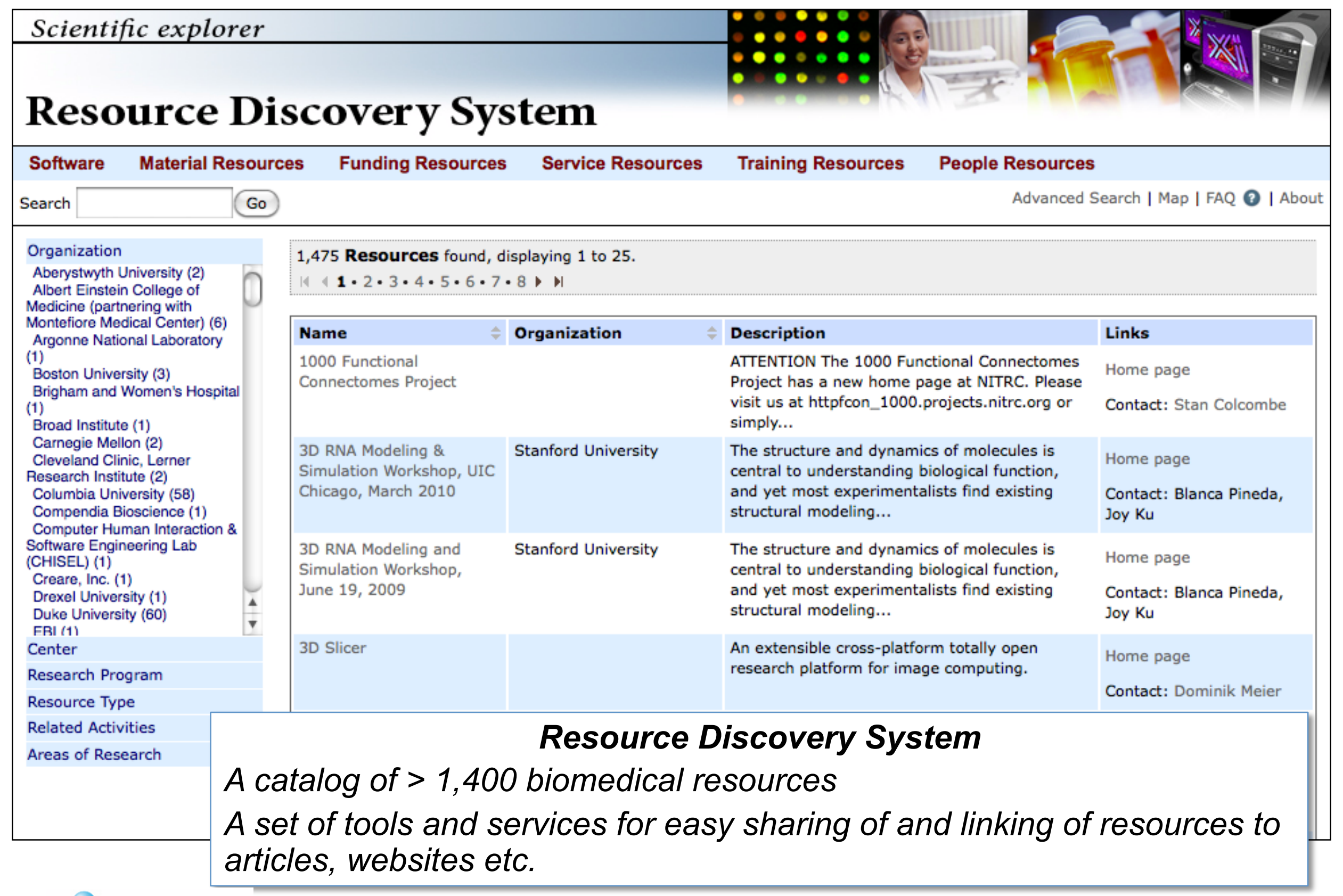

\section{BIOSITEMAPS}




\section{Biositemaps Summary}

- Initially developed in parallel to related efforts initiated by NIF, the CTSAs, and other groups

- Provides a shared framework with which to harmonize access to biomedical resources

- Adopts "Web oriented" philosophy of distributed information without central control

- Uses a formal Biomedical Resource Ontology to enhance precision and recall when searching for resources online 


\section{Acknowledgements}

\section{Contributing Members: Informatics and Translational Supplement}

University of Pittsburgh Stanford University

Michael J. Becich MD PhD

Charles D. Borromeo

Harpreet Singh MS

Nancy B. Whelan

University of Michigan
Brian D. Athey PhD
Aaron Bookvich
Peter Boisvert
Beth Kirschner
Barbara R. Mirel DArts
Paul Saxman MS
Kevin A. Smith MSIS
Zachary Wright MSI

Csongor I. Nyulas MS

Dave Rubenson PhD

Daniel L. Rubin MD MS

Trish Whetzel PhD

\section{Oregon Health \& Science University \\ Shannon McWeeney PhD}
University of Texas HoustonUniversity of California Elmer V. Bernstam MD MSE MS San Diego

\section{Duke University}
Maryann E. Martone PhD
Anita Bandrowski, PhD

Geoffrey S. Ginsburg MD PhD Jessica D. Tenenbaum PhD
Emory University
Tim T. Morris

University of California

Davis

Kent Anderson MS

Davera Gabriel RN

Alice Tarantal PhD
Inventory Resources Working Group Members (38 POCs)

Bill Adams, Rebecca Bamber, Edward Barbour, Teresa Bosler, Jim Brinkley, Christopher Chute, Curtis Cole, Will Digrazio, David Eichmann, Joe Ellefson, Larray Errecary, Sandy Frawley, Steve Johnson, Josef Kalna, Michael Kamerick, Warren Kibbe, Matthew Kristin, Bernie LaSalle, Elliot Leftkowitz, Harold Lehmann, Sandy Mackenzie, Doug McFadden, Rekha Meyer, Marc Overhage, Philip Payne, David Pilasky, Brad Pollock, Mark Porter, Dan Schwartz, Jonathan Silverstein, Rob Taylor, Tom Yeager

\section{Funding Provided by} CTSA

Administrative Supplement (Pittsburgh), 3UL1RR024153-03S1

Administrative Supplement (Duke), 5UL1RR024128-03S1

\section{National Institutes of Health}

Elaine Collier MD

Peter Lyster PhD

\section{NCBC}

National Center for Integrative Biomedical Informatics, 3U54DA021519-04S1

National Center for Biomedical Ontology, 3U54HG004028-04S1

Emory University CTSA, 1UL1RR025008-01

University of California Davis CTSA, 1UL1RR024146-01

University of Michigan CTSA, 1UL1RR024986-01

University of Pittsburgh CTSA, 1UL1RR024153-01

http://biositemaps.ncbcs.org 